\title{
Ethnobotany and Traditional Knowledge of Bamboos (Poaceae: Bambusoideae) in Asia and Their Applications in the Complementary and Alternative Medicine: A Review
}

\author{
Mohammad Amil Zulhilmi Benjamin', Shean Yeaw Ng', Fiffy Hanisdah Saikim', Nor Azizun Rusdi*
}

\section{Mohammad Amil Zulhilmi Benjamin', Shean Yeaw $\mathrm{Ng}^{1}$, Fiffy \\ Hanisdah Saikim', Nor Azizun \\ Rusdi* ${ }^{1 *}$ \\ 'Institute for Tropical Biology and Conservation, Universiti Malaysia Sabah, Jalan UMS, 88400 Kota Kinabalu, Sabah, MALAYSIA.}

\section{Correspondence}

\section{Nor Azizun Rusdi}

Institute for Tropical Biology and

Conservation, Universiti Malaysia Sabah,

Jalan UMS, 88400 Kota Kinabalu, Sabah, MALAYSIA.

E-mail: azizun@ums.edu.my

History

- Submission Date: 07-09-2021;

- Review completed: 28-09-2021;

- Accepted Date: 06-10-2021.

DOI : 10.5530/pj.2021.13.226

Article Available online

http://www.phcogj.com/v13/i6

\section{Copyright}

(c) 2021 Phcogi.Com. This is an openaccess article distributed under the terms of the Creative Commons Attribution 4.0 International license.

\begin{abstract}
Plants are considered a great source of various herbal medicines in the treatment of certain diseases and ailments. There is a growing interest in the utilization of indigenous medicinal plants as the source for complementary and alternative medicine (CAM) due to the significant contribution of plant-based materials to the pharmaceutical field. Bambusoideae is a large grass family of Poaceae, comprising approximately 119 genera and 1482 described species. About $70 \%$ of the bamboo forests are covered throughout Asia. This study aims to provide an informative review of the ethnobotanical significance and traditional knowledge of medicinal plants belonging to the Bambusoideae. This review comprises informative data on medicinal plants, their uses, and parts used by indigenous people and native communities in Asian regions. In line with this review, bamboo has made significant contributions to the ethnobotanical field, specifically as therapeutics for specific diseases. Ethnobotanical data has also made a successful contribution to the CAM. Therefore, the present review on ethnobotany and traditional knowledge of bamboo is expected to have many benefits and could be a good starting point for future work in the pharmaceutical field, both locally and internationally.

Key words: Asia, Bambusoideae, Complementary and alternative medicine, Ethnobotany, Traditional knowledge.
\end{abstract}

\section{INTRODUCTION}

Early plants started colonizing the ground about 450 million years ago and have taken advantage of their versatile metabolism to produce various natural medicinal products. ${ }^{1}$ One common strategy used to identify new drugs is by exploring folk herbal extracts. ${ }^{2}$ The earliest scientific documentation of potential medicinal use of bamboo was published in the early 1960s, ${ }^{3}$ followed by a series of studies conducted by Shibata et al. in the $1970 \mathrm{~s}^{4-8}$ Bamboo, as a biomedical research topic, was relatively silent during the 1980s and 1990s, but research interest has increased worldwide since the beginning of this century. ${ }^{9}$ Thus, bamboo plants play a significant role in traditional Asian medicine over six decades ago.

The family Poaceae (grasses), known as bamboo (or Bambu, a vernacular word of unknown Oriental origin), includes 12 subfamilies, the largest of which is the Bambusoideae, which includes 119 genera and approximately 1482 species. These species are further divided into three tribes: Bambuseae (tropical woody bamboos with 66 genera and 812 species), Arundinarieae (temperate woody bamboos with 31 genera and 546 species), and Olyreae (herbaceous bamboos with 12 genera and 124 species). ${ }^{10-12}$ The molecular phylogenetic results support the recognition of three tribes within the Bambusoideae. ${ }^{11,13}$ These grasses have woody culm with leaves adjusted to support and protect the tender young shoots, an outer ligule (contraligule) on the foliage leaves, gregarious monocarpy (ranging from a few years to 120 years for the flowering cycle), complex vegetative branching, and have bisexual flowers. ${ }^{14-16}$ Herbaceous bamboos typically lack differentiated outer ligules and culm leaves, unisexual spikelets, and have confined vegetative branching. ${ }^{14,16}$ Excluding the New Guinea endemic Buergersiochloa, all Olyreae have crenate (olyroid) silica bodies. ${ }^{17-19}$

According to the Food and Agriculture Organization $(\mathrm{FAO}),{ }^{20}$ Asia contributes to the world's largest source of bamboo resources. However, there is no comprehensive and up-to-date checklist of plants, particularly the medicinal plants of Asian regions. Notably, Asia and Pacific regions account for about $80 \%$ of bamboo forests and species available worldwide. ${ }^{21}$ Within its temperate and tropical climates, Asia has many advantages for bamboo cultivation, accounting for more than 900 species in 40-50 genera. Many Asian countries such as China, India, Thailand, Indonesia, Vietnam, and Myanmar are rich in bamboo resources. ${ }^{22}$ As a result, this review article focuses on the ethnobotanical and traditional knowledge of medicinal plants in the Bambusoideae specifically used by indigenous people and native communities in Asian regions. The medicinal plants, together with their uses, are reviewed accordingly throughout this article. Likewise, the applications of complementary and alternative medicine (CAM) are also addressed.

\section{BACKGROUND OF BAMBOOS IN ASIAN REGIONS}

Bamboos are known to be durable and fast-growing with short harvest periods. Furthermore, they are sustainable (in terms of yield), inexpensive, and have 
supported livelihoods throughout history. These factors make bamboo a reliable resource for meeting the needs of an increasing population, market, and development. ${ }^{23-24}$ Thus, it has been so intricately related to the livelihood of Asia people, representing the bamboo civilization on the continent. ${ }^{25}$ These grasses are found throughout Africa, Latin America, and Asia, but their origins can be traced back to Southeast Asia. Bamboo's geographical distribution is generally determined by climates. ${ }^{26}$ It is widely distributed in the tropical and subtropical zones approximately between $46^{\circ} \mathrm{N}$ and $47^{\circ} \mathrm{S}$ latitude, reaching elevations as high as $4000 \mathrm{~m}$ in the Himalayas and parts of China as it adapts easily to a variety of climatic and soil conditions. ${ }^{27-28}$ Bamboo thrives in temperatures ranging from 8.8 to $36^{\circ} \mathrm{C},{ }^{29}$ but some species can still grow in colder climates with temperatures as low as $-20^{\circ} \mathrm{C} .{ }^{30} \mathrm{Bamboo}$ grows best in areas with high rainfall, ranging from $1270 \mathrm{~mm}$ to 6350 $\mathrm{mm}$ or more, ${ }^{31-32}$ thus highlighting the significant impact of rainfall on the distribution and growth of these species.

As shown in Figure 1, the Asian-Pacific Region (I), the American Region (II), and Africa (III) are the main producing areas of bamboo. Around $80 \%$ of the world's bamboo forest is located in the Asian-Pacific regions, ${ }^{21}$ whereas India and China account for approximately $70 \%$ of Asia's bamboo forests. ${ }^{22}$ Along with a recent report, Asia is the world's largest source of bamboo resources. ${ }^{20}$ China has the most bamboo species (626 species), ${ }^{33}$ followed by India, which has 115 species. ${ }^{34}$ Surprisingly, about $25 \%$ of all bamboo species were discovered in India, particularly in the biodiversity hotspot regions like northeast India and western Ghats. ${ }^{35-36}$ Bamboo fossil records in Asia suggest that bamboo was most likely dispersed from India following the collision of the Eurasian Plate with the Indian Plate. Nevertheless, more fossil records from Asia are required to validate this dispersal route because another from Europe cannot be ruled out. ${ }^{37}$

More than two million tonnes of bamboo shoots are consumed in Asia annually. Since ancient times, bamboo products have been consumed either as fresh, dried, canned, boiled, fermented, or as medicine. These practices are common in many Asian countries, including Bhutan, China, Indonesia, Japan, Korea, Malaysia, Nepal, and northeast India. ${ }^{12,38-40}$ Both fresh and fermented bamboo shoots are crucial sources of income for rural communities because they are high in fat, carbohydrates, protein, minerals, vitamins, enzymes, coenzymes, reducing and non-reducing sugars, and lactic acid (if fermented). ${ }^{41-49}$ Thus, they have been widely used as a source of nutraceutical products. For instance, underground shoots of bamboo are cooked as a vegetable soup ingredient called laswa in the Philippines. ${ }^{50}$ Bamboo salts (jookyeom) are roasted with regular salt, yellow clay, and bamboo culms in Korean folk medicine to treat chronic diseases. ${ }^{51-53}$ Tender shoots of a few species are consumed in India as pickles, vegetables, and curries. ${ }^{43,48,54}$ In most bamboo-growing countries, clean young bamboo leaves are dried, roasted, and consumed as tea. It is considered a tasty and healthy beverage..$^{40}$

\section{ETHNOBOTANY AND TRADITIONAL KNOWL- EDGE OF BAMBOOS IN ASIA}

In 1896, American botanist John Harshberger coined the term ethnobotany, which refers to "the study of plants used by humans". ${ }^{55}$ Ethnobotany is a branch of botany that focuses on products derived from natural sources such as food, coloring agents, fiber plants, fertilizers, building materials, dyes, tan, taboos, avoidance, magico-religious beliefs about plants, and other functional and harmful plants. ${ }^{56-58} \mathrm{In}$ other words, ethnobotany is the study of how people in specific regions and cultures use indigenous plants. ${ }^{59}$ It is now widely acknowledged that ethnobotany is closely tied with the natural and traditional relationship between plants and humans in a dynamic ecosystem. Ethnic people have a strong belief in native folklore medicine as part of the system and rely heavily on plants for almost all of their daily needs and requirements.

Traditional medicine is a collection of empirical practices that are inextricably associated with the religious practices and beliefs of indigenous cultures. Traditional medicine relies heavily on medicinal plant knowledge. According to the World Health Organization, roughly $80 \%$ of the world's population (six billion people) depends primarily on traditional medicines. ${ }^{60}$ In addition, anthropological research has been extensively conducted. ${ }^{61-62}$ However, traditional medicine is widely used in modern society despite the archaeological record's remarkable lack of visibility of such medicine. This review article discusses the ethnobotanical parts and traditional knowledge of medicinal plants from the Bambusoideae used by indigenous people and native communities in Asian countries. Based on Table 1, the medicinal properties of bamboo plants and their applications are discussed throughout this article.

\section{APPLICATION OF BAMBOOS IN THE COMPLE- MENTARY AND ALTERNATIVE MEDICINE}

For Millennia, Bamboos have been utilized in traditional Asian medicine, particularly in China and India. ${ }^{115-116}$ In folk medicine, all parts of this plant are used. Cough, fever, leprosy, hypertension, lung inflammation, cardiovascular diseases, arteriosclerosis, osteoarthritis, and osteoporosis are all treated with the leaves. ${ }^{115,117-118}$ In addition to being a food source, primarily in Asian countries, bamboo shoots are used to cure and prevent cancer and cardiovascular complications,

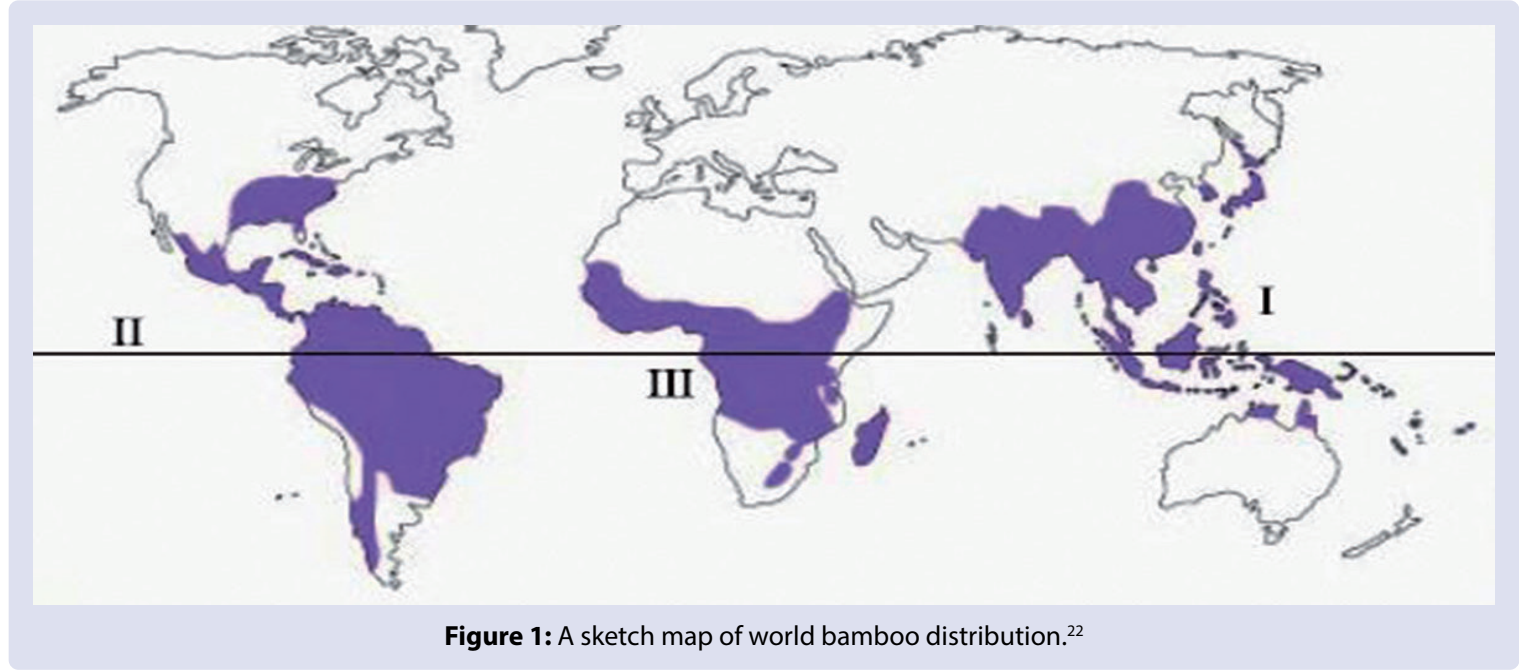


Benjamin MAZ, et al:: Ethnobotany and Traditional Knowledge of Bamboos (Poaceae: Bambusoideae) in Asia and Their Applications in the Complementary and Alternative Medicine: A Review

Table 1: Summary of bamboos as medicinal plants according to Asian countries.

\begin{tabular}{|c|c|c|c|c|c|c|c|}
\hline Scientific names & $\begin{array}{l}\text { Common } \\
\text { names }\end{array}$ & Parts used & Preparation & Application & Ailments treated & Places & References \\
\hline \multicolumn{8}{|l|}{ Bangladesh } \\
\hline \multirow{2}{*}{$\begin{array}{l}\text { Bambusa multiplex } \\
\text { (Lour.) Raeusch. ex } \\
\text { Schult.f. }\end{array}$} & \multirow[b]{2}{*}{ Thirwa } & Leaves & Juice & Oral & Fevers & \multirow{4}{*}{$\begin{array}{l}\text { Chittagong Hill } \\
\text { Tracts }\end{array}$} & \multirow{4}{*}[63]{} \\
\hline & & Roots & Paste & Topical & Abscesses, itches & & \\
\hline \multirow{2}{*}{$\begin{array}{l}\text { Bambusa oldhamii } \\
\text { Munro }\end{array}$} & \multirow{2}{*}{ O-anna } & Leaves & Juice & Oral & Fevers & & \\
\hline & & Roots & Paste & Topical & Abscesses, itches & & \\
\hline Bambusa tulda Roxb. & Bans & $\begin{array}{l}\text { Soft inner core of } \\
\text { stems }\end{array}$ & $\begin{array}{l}\text { Decoction (with } \\
\text { Terminalia belerica sliced } \\
\text { fruits, Terminalia chebula } \\
\text { fruits, Phyllanthus } \\
\text { emblica fruits, Solanum } \\
\text { violaceum sliced leaves, } \\
\text { and Drynaia quercifolia } \\
\text { roots }\end{array}$ & Oral & $\begin{array}{l}\text { Cardiovascular disorders, } \\
\text { weakness of heart }\end{array}$ & $\begin{array}{l}\text { Muktipara, } \\
\text { Chuadanga Sadar, } \\
\text { Chuandanga } \\
\text { District }\end{array}$ & {$[64]$} \\
\hline $\begin{array}{l}\text { Bambusa bambos (L.) } \\
\text { Voss }\end{array}$ & Kanta Bans & $\begin{array}{l}\text { Stems, leaves, } \\
\text { roots, sprouts, } \\
\text { barks }\end{array}$ & - & - & $\begin{array}{l}\text { Laxative, leukoderma, } \\
\text { inflammation, strangury, } \\
\text { cough, cold, consumption, } \\
\text { asthma, emmenagogue, } \\
\text { bleeding disorder }\end{array}$ & $\begin{array}{l}\text { Rangamati } \\
\text { Hill District, } \\
\text { Chittagong Hill } \\
\text { Tracts }\end{array}$ & {$[65]$} \\
\hline Bambusa tulda Roxb. & Mitinga & Leaves & & & Arthritis, rheumatism & & \\
\hline $\begin{array}{l}\text { Bambusa arundinacea } \\
\text { (Retz.) Willd. }\end{array}$ & Bans & $\begin{array}{l}\text { Stems } \\
\text { Leaves } \\
\text { Roots }\end{array}$ & - & - & $\begin{array}{l}\text { Leukoderma } \\
\text { Cough, cold } \\
\text { Joint pains }\end{array}$ & Kushtia District & {$[66]$} \\
\hline \multicolumn{8}{|l|}{ Cambodia } \\
\hline Bambusa spp. & $\begin{array}{l}\text { Russey Srok, } \\
\text { Russey Prey }\end{array}$ & Woods & Decoction & Oral & Liver disorders & $\begin{array}{l}\text { Phnom Penh; } \\
\text { Kandal; Takeo; } \\
\text { Kampong Speu }\end{array}$ & {$[67]$} \\
\hline \multicolumn{8}{|l|}{ China } \\
\hline $\begin{array}{l}\text { Phyllostachys nigra } \\
\text { (Lodd. ex Lindl.) } \\
\text { Munro }\end{array}$ & - & - & - & - & - & $\begin{array}{l}\text { Chuxiong, } \\
\text { Central Yunnan }\end{array}$ & {$[68]$} \\
\hline $\begin{array}{l}\text { Bambusa pervariabilis } \\
\text { McClure }\end{array}$ & Zhu Ru & Shavings & - & - & $\begin{array}{l}\text { Clearing heat, stopping } \\
\text { bleeding }\end{array}$ & Sansui County & [69] \\
\hline \multirow{3}{*}{$\begin{array}{l}\text { Dendrocalamus } \\
\text { hamiltonii Nees \& Arn. } \\
\text { ex Munro }\end{array}$} & \multirow{4}{*}{ Aqqyul } & $\begin{array}{l}\text { Water inside the } \\
\text { culms }\end{array}$ & Burnt & Oral & Fever & \multirow{8}{*}{$\begin{array}{l}\text { Xishuangbanna, } \\
\text { Yunnan Province }\end{array}$} & \multirow{8}{*}[70]{} \\
\hline & & Culms & $\begin{array}{l}\text { Boiled with ginger and } \\
\text { garlic }\end{array}$ & Ural & Food poisoning & & \\
\hline & & Culm & - & - & Oedema & & \\
\hline $\begin{array}{l}\text { Bambusa vulgaris } \\
\text { Schrad. ex J.C. Wendl. }\end{array}$ & & - & $\begin{array}{l}\text { Boiled with Nicolsonia } \\
\text { renifolia, Dillenia indica, } \\
\text { and Mahonia spp. }\end{array}$ & $\begin{array}{l}\text { Taken with } \\
\text { sugar and } \\
\text { rice }\end{array}$ & Hepatitis & & \\
\hline $\begin{array}{l}\text { Gigantochloa albociliata } \\
\text { Munro }\end{array}$ & & Roots, buds & Decoction & Oral & $\begin{array}{l}\text { Dysmenorrhea, irregular } \\
\text { menstruation, postpartum } \\
\text { blood clots }\end{array}$ & & \\
\hline $\begin{array}{l}\text { Phyllostachys nigra } \\
\text { (Lodd. ex Lindl.) } \\
\text { Munro }\end{array}$ & - & Leaves & - & - & Malaria & & \\
\hline $\begin{array}{l}\text { Schizostachyum } \\
\text { funghomii McClure }\end{array}$ & & Nodes & $\begin{array}{l}\text { Decoction with Areca } \\
\text { catechu petioles }\end{array}$ & & $\begin{array}{l}\text { Headache, body ache, } \\
\text { common cold, increase } \\
\text { appetite }\end{array}$ & & \\
\hline Dendrocalamus spp. & & Roots & $\begin{array}{l}\text { Decoction with leaves } \\
\text { of Areca catechu fruit } \\
\text { or Alpinia galanga, } \\
\text { Gendarussa vulgaris } \\
\text { roots, and Adhatoda } \\
\text { vasica roots }\end{array}$ & Oral & Induce urination & & \\
\hline
\end{tabular}


Benjamin MAZ, et al.: Ethnobotany and Traditional Knowledge of Bamboos (Poaceae: Bambusoideae) in Asia and Their Applications in the Complementary and Alternative Medicine: A Review

\begin{tabular}{|c|c|c|c|c|c|c|c|}
\hline Fargesia spp. & & $\begin{array}{l}\text { Red bamboo } \\
\text { fungus }\end{array}$ & & & Skin inflammation & & \\
\hline $\begin{array}{l}\text { Indosasa pingbianensis } \\
\text { McClure }\end{array}$ & & Shoots & & & Common cold, headache & & \\
\hline $\begin{array}{l}\text { Phyllostachys glauca } \\
\text { McClure }\end{array}$ & - & Leaves & - & - & Cough, lung inflammation & Yunnan & {$[71]$} \\
\hline $\begin{array}{l}\text { Phyllostachys } \\
\text { heterocycla (Carrière) } \\
\text { Matsum. }\end{array}$ & & $\begin{array}{l}\text { Sap of young } \\
\text { culms }\end{array}$ & & & Cough, throat inflammation & & \\
\hline \multicolumn{8}{|l|}{ India } \\
\hline $\begin{array}{l}\text { Bambusa arundinacea } \\
\text { (Retz.) Willd. }\end{array}$ & Moongil & $\begin{array}{l}\text { Young leaves, } \\
\text { terminal buds } \\
\text { (with turmeric } \\
\text { leaves) }\end{array}$ & $\begin{array}{l}\text { Grounded with } \\
\text { Aloe vera, paste }\end{array}$ & Topical & Fractured bones & $\begin{array}{l}\text { Pudukottai } \\
\text { District, Tamil } \\
\text { Nadu }\end{array}$ & {$[72]$} \\
\hline $\begin{array}{l}\text { Bambusa arundinacea } \\
\text { (Retz.) Willd. }\end{array}$ & Moongil & Seeds, seed oil & Paste & Oral & Rheumatism & $\begin{array}{l}\text { Tirunelveli Hills } \\
\text { of Western Ghats }\end{array}$ & {$[73]$} \\
\hline $\begin{array}{l}\text { Bambusa arundinacea } \\
\text { (Retz.) Willd. }\end{array}$ & Baans & Roots & Decoction & Oral & Kidney stone & $\begin{array}{l}\text { Udhampur } \\
\text { District of Jammu } \\
\text { and Kashmir }\end{array}$ & {$[74]$} \\
\hline $\begin{array}{l}\text { Bambusa vulgaris } \\
\text { Schrad. ex J.C. Wendl. }\end{array}$ & - & - & - & - & Type 2 diabetes & $\begin{array}{l}\text { Tiruvallur } \\
\text { District, Tamil } \\
\text { Nadu }\end{array}$ & {$[75]$} \\
\hline $\begin{array}{l}\text { Dendrocalamus strictus } \\
\text { (Roxb.) Nees }\end{array}$ & Math & Gums & $\begin{array}{l}\text { Soaked in water } \\
\text { Powdered }\end{array}$ & $\begin{array}{l}\text { Topical in } \\
\text { ear } \\
\text { Oral with } \\
\text { water }\end{array}$ & $\begin{array}{l}\text { Ear ache } \\
\text { Tuberculosis }\end{array}$ & $\begin{array}{l}\text { Amravati District, } \\
\text { Maharashtra }\end{array}$ & {$[76]$} \\
\hline Bambusa sp. & Bans & $\begin{array}{l}\text { Leaves } \\
\text { Barks } \\
\text { Seeds }\end{array}$ & $\begin{array}{l}\text { Juice } \\
\text { Decoction } \\
\text { Mixed with Shahad } \\
\text { (honey) }\end{array}$ & Oral & Cancer & Chhattisgarh & {$[77]$} \\
\hline $\begin{array}{l}\text { Dendrocalamus strictus } \\
\text { (Roxb.) Nees }\end{array}$ & Bans & Leaves & Decoction & - & Clear uterus & $\begin{array}{l}\text { Chittorgarh } \\
\text { District, } \\
\text { Rajasthan }\end{array}$ & {$[78-79]$} \\
\hline $\begin{array}{l}\text { Bambusa arundinacea } \\
\text { (Retz.) Willd. }\end{array}$ & Mungil & Leaves & Juice (mixed with honey) & Oral & Cough in children & $\begin{array}{l}\text { Atoor Village, } \\
\text { Kanyakumari } \\
\text { District, Tamil } \\
\text { Nadu }\end{array}$ & {$[80]$} \\
\hline $\begin{array}{l}\text { Bambusa arundinacea } \\
\text { (Retz.) Willd. }\end{array}$ & Mulmunkil & $\begin{array}{l}\text { Roots } \\
\text { Leaves }\end{array}$ & $\begin{array}{l}\text { Decoction } \\
\text { Infusion }\end{array}$ & - & Skin diseases & $\begin{array}{l}\text { Kanyakumari } \\
\text { District, Tamil } \\
\text { Nadu }\end{array}$ & {$[81]$} \\
\hline Bambusa balcooa Roxb. & - & - & - & - & - & Tripura & {$[82]$} \\
\hline $\begin{array}{l}\text { Dendrocalamus } \\
\text { giganteus Munro }\end{array}$ & $\begin{array}{l}\text { Wa Mishel, } \\
\text { Kepaii/Mpin }\end{array}$ & Fresh culms & Scrapped (wool made) & Topical & Fresh cuts and wounds & $\begin{array}{l}\text { North Cachar } \\
\text { Hills of Assam }\end{array}$ & {$[83]$} \\
\hline $\begin{array}{l}\text { Bambusa arundinacea } \\
\text { (Retz.) Willd. }\end{array}$ & Moongil & Seeds & - & - & Rheumatism & $\begin{array}{l}\text { Pachamalai Hills } \\
\text { of Tamil Nadu }\end{array}$ & {$[84]$} \\
\hline $\begin{array}{l}\text { Bambusa vulgaris } \\
\text { Schrad. ex J.C. Wendl. }\end{array}$ & Mughil & Leaves & Extracted & Oral & Reduced sperm count & $\begin{array}{l}\text { Kathiyavadi } \\
\text { Village, Vellore } \\
\text { District, Tamil } \\
\text { Nadu }\end{array}$ & {$[85]$} \\
\hline $\begin{array}{l}\text { Bambusa arundinacea } \\
\text { (Retz.) Willd. }\end{array}$ & Veduru & Leaves & Decoction & Oral & Asthma & Andhra Pradesh & {$[86]$} \\
\hline Bambusa tulda Roxb. & Bans & Roots & Decoction & Oral & Piles, constipation & Assam & {$[87]$} \\
\hline Bambusa tulda Roxb. & Ejo & & & & Tetanus infection & & \\
\hline $\begin{array}{l}\text { Dendrocalamus } \\
\text { giganteus Munro }\end{array}$ & & - & - & & Steroid drug & & \\
\hline $\begin{array}{l}\text { Dendrocalamus strictus } \\
\text { (Roxb.) Nees }\end{array}$ & - & $\begin{array}{l}\text { Siliceous matter } \\
\text { Leaves }\end{array}$ & Decoction & - & $\begin{array}{l}\text { Astringent, tonic } \\
\text { Abortifacient }\end{array}$ & $\begin{array}{l}\text { Arunachal } \\
\text { Pradesh }\end{array}$ & {$[88]$} \\
\hline $\begin{array}{l}\text { Schizostachyum } \\
\text { capitatum (Munro) } \\
\text { R.B.Majumdar }\end{array}$ & & Leaves & Infusion & & Stomach pain & & \\
\hline $\begin{array}{l}\text { Bambusa bambos (L.) } \\
\text { Voss }\end{array}$ & Moongil & Seeds & Paste & Topical & Rheumatism & $\begin{array}{l}\text { Palamalai Region } \\
\text { of Eastern Ghats }\end{array}$ & [89] \\
\hline
\end{tabular}




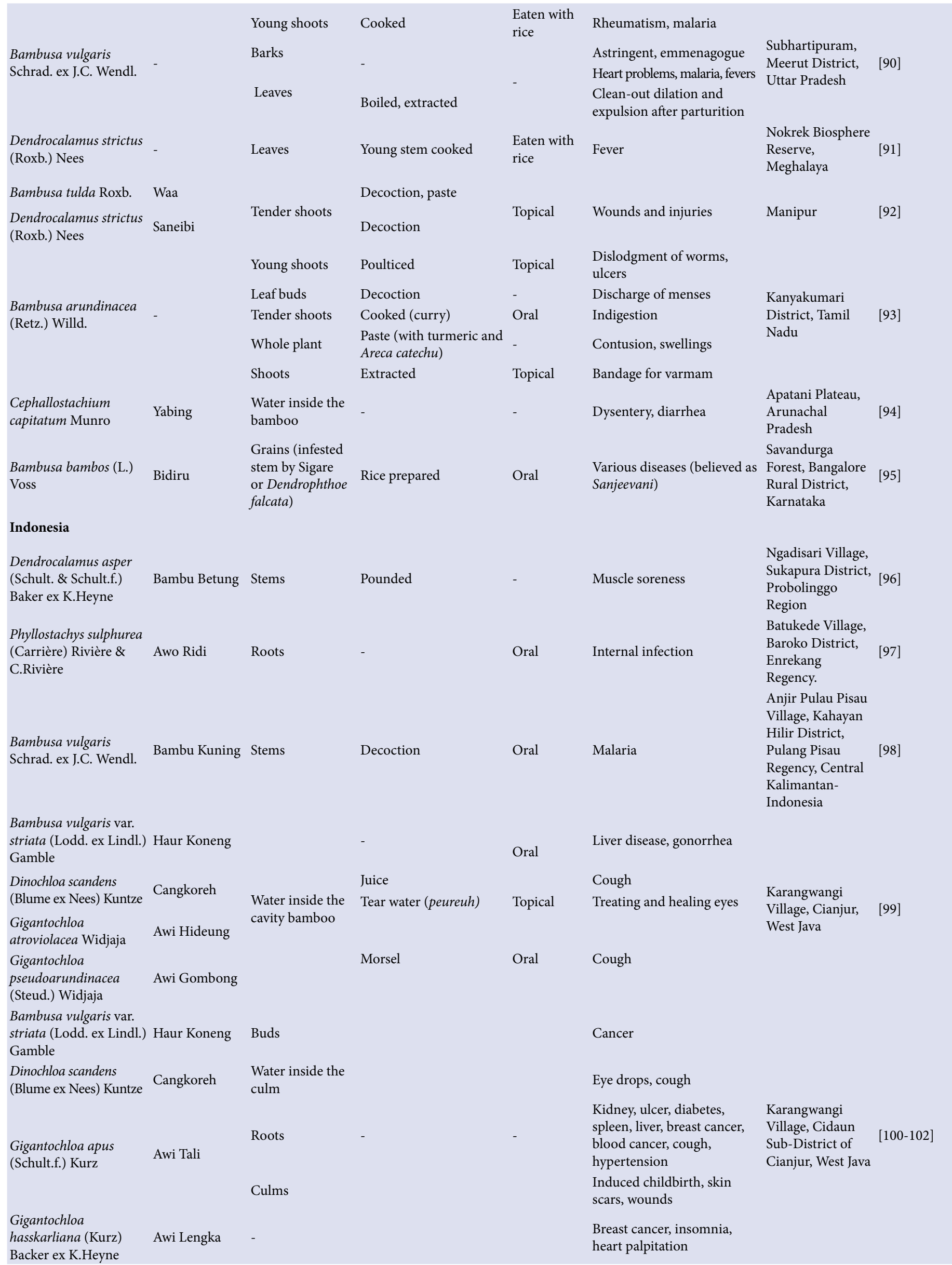




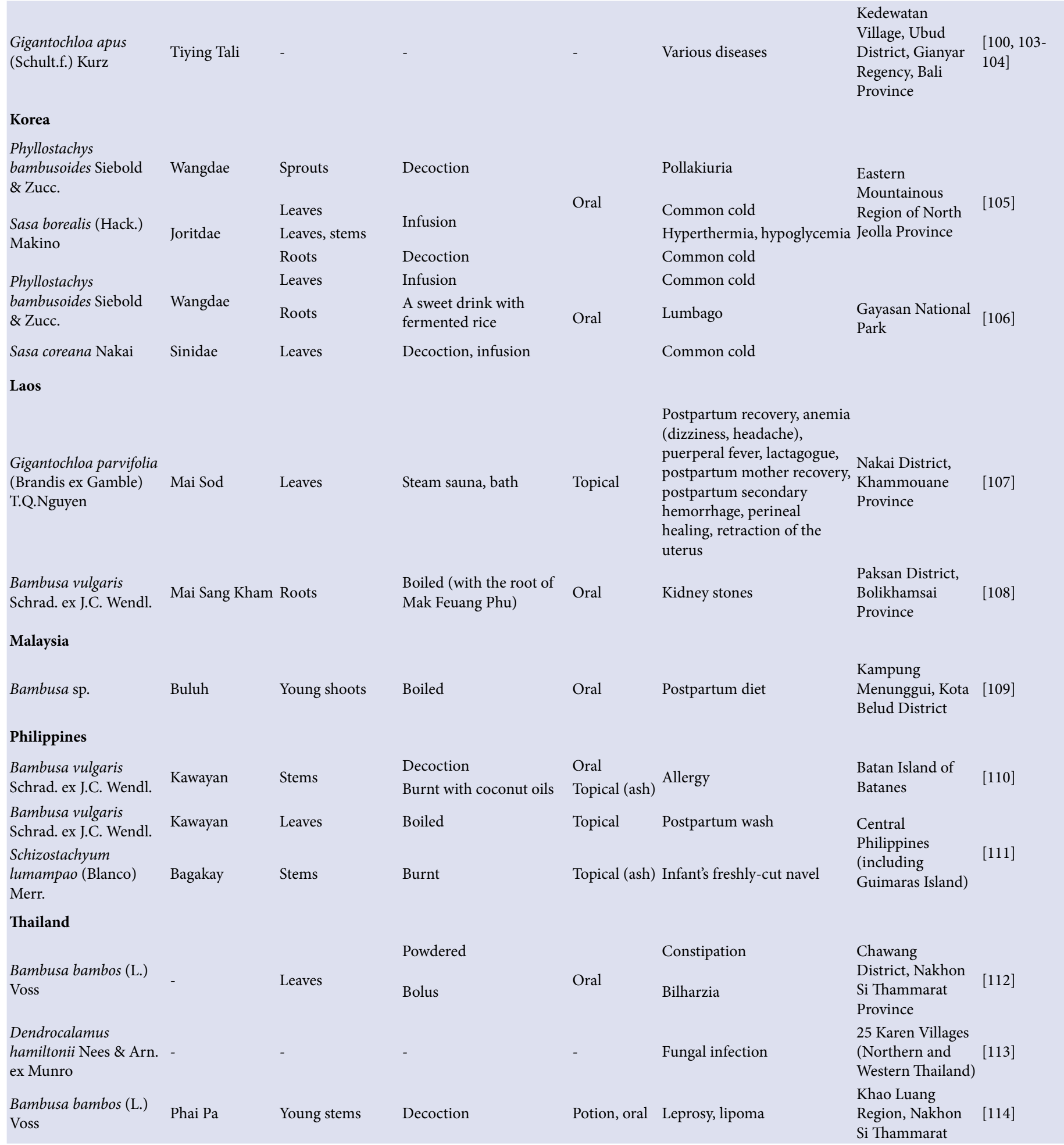


Table 2: Bamboos in traditional medicinal systems. ${ }^{125}$

\begin{tabular}{|c|c|c|}
\hline Name & Constituents & Health benefits \\
\hline \multicolumn{3}{|c|}{ Ayurveda, Tibetan, and Unani Traditional Medicines } \\
\hline Tabasheer & $\begin{array}{l}\text { Siliceous secretion is often called bamboo silica or bamboo-manna found in } \\
\text { the hollow internodes of various species of bamboos. It may be transparent, } \\
\text { translucent, chalky, or mainly composed of silicic acid (up to } 96.9 \% \text { ) with } \\
\text { organic matter above } 1 \% \text {. }\end{array}$ & $\begin{array}{l}\text { It acts as an antispasmodic, aphrodisiac, astringent, cooling } \\
\text { tonic, cough, febrifuge, stimulant, and relieves asthma. }\end{array}$ \\
\hline Sitopaladi Churna & $\begin{array}{l}\text { Powder made with tabasheer as the main ingredient, plus small amounts of } \\
\text { long pepper, cardamom, and cinnamon in the base of sugar. }\end{array}$ & $\begin{array}{l}\text { A popular remedy for common colds, coughs, sinus } \\
\text { congestion, sore throats, tuberculosis, and other lung diseases. }\end{array}$ \\
\hline \multicolumn{3}{|c|}{ Traditional Chinese Medicine } \\
\hline Chenjin Wan & $\begin{array}{l}\text { Arisaema, Bamboo shaving and Tabasheer, Chrysanthemum, Citrus, } \\
\text { Fritillaria, Ophiopogon, Salvia, apricot seed, biota, ginger, hoelen, silkworm. }\end{array}$ & $\begin{array}{l}\text { Phlegm mist obstructing the orifices yields symptoms of } \\
\text { blurred vision, insomnia, and restlessness. }\end{array}$ \\
\hline Gualou Zhishi Tang & $\begin{array}{l}\text { Bamboo shaving and sap, Citrus, Fritillaria, Gardenia, Platycodon, } \\
\text { Saussurea, Trichosanthes seed, chih-shih, licorice, scute, etc. }\end{array}$ & For reducing thick phlegm that is hard to expectorate. \\
\hline Jupi Zhuru Tang & Bamboo shavings, Citrus, Pinellia, hoelen, licorice. & Relieving phlegm. \\
\hline Qinghuo Ditan Tang & $\begin{array}{l}\text { Arisaema, Bamboo shaving and Tabasheer, Chrysanthemum, Citrus, } \\
\text { Fritillaria, Ophiopogon, Salvia, apricot seed, biota, ginger, hoelen, silkworm. }\end{array}$ & $\begin{array}{l}\text { Phlegm mist obstructing the orifices yields symptoms of } \\
\text { blurred vision, insomnia, and restlessness. }\end{array}$ \\
\hline Qinggong Tang & $\begin{array}{l}\text { Bamboo leaf, Forsythia, Ophiopogon, Scrophularia, lotus plumule, rhino } \\
\text { horn. }\end{array}$ & $\begin{array}{l}\text { Fever with dryness, penetrating to the pericardium, with } \\
\text { delirium }\end{array}$ \\
\hline Qingluo Yin & Bamboo leaf, Dolichos flower, Lonicera, Luffa, Mirabilitum, lotus leaf. & Fever with light-headedness, blurry vision, or headaches. \\
\hline Xiaoer Qizhen Dan & Arisaema, Cinnabar, Tabasheer, realgar. & For coughing, phlegm, and wheezing. \\
\hline Zhuye Shigao Tang & Bamboo leaf, Ophiopogon, Oryza, Pinellia, ginseng, gypsum, licorice. & Fever with dryness, insomnia, and irritability. \\
\hline
\end{tabular}

improve digestion, and treat jaundice. ${ }^{119-120}$ They are also used to treat epilepsy, dysentery, diabetes, respiratory problems, bacterial infections, nervous system disorders, skin ulcers, and as a diuretic. ${ }^{24,117}$

Complementary and alternative medicine (CAM) is described as "healthcare and medical practices that are not currently an integral part of conventional medicine". ${ }^{121}$ Herbal drugs, traditional medicines, meditation, and religious ceremonies are cases of such practices. More than $40 \%$ of western populations practice CAM for a variety of health conditions. ${ }^{122}$ Interestingly, Bambusa sp. stem was discovered to be used as a CAM by diabetes mellitus patients. ${ }^{123}$ Due to its connection with Traditional Chinese Medicine (TCM), the findings of moso bamboo (Phyllostachys edulis) in treating diabetes mellitus are also being further investigated. ${ }^{124}$ As a result, Asian bamboo species are widely used for medicinal purposes, implying that they could be a CAM in the future due to the uniqueness of their ethnobotany connections. Some lists of bamboo products or parts that were used as CAM are presented in Table 2.

\section{CONCLUSION}

In conclusion, plants belonging to the Bambusoideae have contributed massively to ethnobotanical and pharmacological fields. This review article summarized bamboo plants that have been used by indigenous people and native communities in Asian countries, specifically to treat certain diseases and ailments. Interestingly, these documented plant species, together with their medicinal values, could be a good starting point for future work in the CAM. In order to prevent the destruction of plant resources and materials, it is vital to correctly identify them for proper harvesting, as well as the chemicals extracted. Specifically, it is crucial to ensure the correct raw materials are being harvested and supplied for pharmaceutical use, thereby facilitating the conservation and utilization of indigenous medicinal plants in the future. In other words, people are aware of and value indigenous knowledge and heritage. Hence, it is proven that ethnobotanical study could be one of the useful tools to document and record potential medicinal plants, including Bambusoideae, especially in the Asian regions.

\section{ACKNOWLEDGEMENT}

The authors wish to express their appreciation to Universiti Malaysia Sabah for financial support from Postgraduate Research Grant (UMSGreat) [GUG0507-2/2020] during the research period. A sincere appreciation is also dedicated to Sabah Parks and Sabah Biodiversity Centre for providing an access license [JKM/MBS.1000-2/2 JLD.12 (89)].

\section{REFERENCES}

1. Nürnberger T, Brunner F. Innate immunity in plants and animals: emerging parallels between the recognition of general elicitors and pathogen-associated molecular patterns. Curr Opin Plant Biol. 2002;5(4):318-24.

2. Katiyar C, Gupta A, Kanjilal S, Katiyar S. Drug discovery from plant sources: an integrated approach. Ayu. 2012;33(1):10-9.

3. Sakai S, Saito G, Sugayama J, Kamasuka T, Takada S, Takano T. [On the anticancer action of bamboo extract]. J Antibiot. 1963;16:38791.

4. Okabe S, Takeuchi K, Takagi K, Shibata M. Stimulatory effect of the water extract of bamboo grass (Folin solution) on gastric acid secretion in pylorus-ligated rats. Jpn J Pharmacol. 1975;25(5):6089.

5. Shibata M, Yamatake Y, Sakamoto M, Kanamori M, Takagi K. [Phamacological studies on bamboo grass (1). Acute toxicity and anti-inflammatory and antiulcerogenic activities of water-soluble fraction (Folin) extracted from Sasa albomarginata Makino et Shibata]. Folia Pharmacol Jpn. 1975;71(5):481-90.

6. Shibata M, Kubo K, Onoda M. [Pharmacological studies on bamboo grass. (2) Central depressant and antitoxic actions of a watersoluble fraction (Folin) extracted from Sasa albomarginata Makino et Shibata]. Folia Pharmacol Jpn. 1976;72(5):531-41.

7. Shibata M, Kubo K, Onoda M. [Pharmacological studies on bamboo grass. III. Effects on cardiovascular and isolated organs of watersoluble fraction extracted from Sasa albomarginata Makino et Shibata (Bambusaceae)]. Yakugaku Zasshi. 1978;98(10):1436-40.

8. Shibata M, Fujii M, Yamaguchi R. [Pharmacological studies on bamboo grass. IV. Toxicological and pharmacological effects of the extract (FIII) obtained from Sasa albomarginata Makino et Shibata]. Yakugaku Zasshi 1979;99(6):663-8.

9. Panee J. Bamboo extract in the prevention of diabetes and breast cancer. In: Watson RR, editor. Complementary and alternative therapies and the aging population. San Diego: Academic Press; 2009. p. 159-77

10. McClure FA. A glossary of the bamboos. Taxon. 1966;15(6):220-35 
11. Sungkaew S, Stapleton CMA, Salamin N, Hodkinson TR. Nonmonophyly of the woody bamboos (Bambuseae; Poaceae): a multigene region phylogenetic analysis of Bambusoideae s.s. J Plant Res. 2009;122(1):95-108.

12. Clark LG, Londoño X, Ruiz-Sanchez E. Bamboo taxonomy and habitat. In: Liese W, Köhl M, editors. Bamboo: the plant and its uses. Cham: Springer; 2015. p. 1-30.

13. Bouchenak-Khelladi Y, Salamin N, Savolainen V, Forest F, van der Bank M, Chase MW, et al. Large multi-gene phylogenetic trees of the grasses (Poaceae): progress towards complete tribal and generic level sampling. Mol Phylogenet Evol. 2008;47(2):488-505

14. Judziewicz EJ, Clark LG, Londoño X, Stern MJ. American bamboos. Washington: Smithsonian Institution Press; 1999.

15. Grass Phylogeny Working Group. Phylogeny and subfamilia classification of the grasses (Poaceae). Ann Missouri Bot Gard. 2001;88(3):373-457

16. Judziewicz EJ, Clark LG. Classification and biogeography of new world grasses: Anomochlooideae, Pharoideae, Ehrhartoideae, and Bambusoideae. Aliso A J Syst Evol Bot. 2007;23(1):303-14

17. Soderstrom TR, Ellis RP. The position of bamboo genera and allies in a system of grass classification. In: Soderstrom TR, Hilu KW, Campbell CS, Barkworth ME, editors. Grass systematics and evolution. Washington: Smithsonian Institution Press; 1987. p. 225-38.

18. Zhang W, Clark LG. Phylogeny and classification of the Bambusoideae (Poaceae). In: Jacobs SWL, Everett J, editors. Grasses: systematics and evolution. Melbourne: CSIRO Publishing; 2000. p. 35-42.

19. Clark LG, Dransfield S, Triplett J, Sánchez-Ken JG. Phylogenetic relationships among the one-flowered, determinate genera of Bambuseae (Poaceae: Bambusoideae). Aliso A J Syst Evol Bot. 2007;23(1):315-32

20. Food and Agriculture Organization. Global forest resources assessment 2010: main report. Rome: FAO Forestry Paper 163; 2010.

21. Fu M, Xiao J, Lou Y. Cultivation and utilization on bamboo. Beijing China Forestry Publishing House; 2000.

22. Mera FAT, Xu C. Plantation management and bamboo resource economics in China. Ciencia y Tecnología. 2014;7(1):1-12.

23. Jijeesh CM, Seethalakshmi KK. Potential role of bamboo plantations in afforestation/reforestation CDM (Clean Development Mechanism). Evergreen. 2009;61/62:8-10.

24. Honfo $H$, Tovissodé FC, Gnanglè C, Mensah S, Salako VK, Assogbadjo $A E$, et al. Traditional knowledge and use value of bamboo in southeastern Benin: implications for sustainable management. Ethnobot Res Appl. 2015;14:139-53

25. Nath AJ, Lal R, Das AK. Managing woody bamboos for carbon farming and carbon trading. Glob Ecol Conserv. 2015;3:654-63.

26. Gamble JS. The Bambuseae of British India. Vol 7. Calcutta: Printed at the Bengal Secretariat Press; 1896.

27. Dransfield S. The bamboos of Sabah. Sabah: Forestry Department; 1992

28. McNeely JA. Bamboo, biodiversity and conservation in Asia. In: Ramanatha Rao V, Ramanuja Rao IV, editors. Biodiversity and genetic conservation. Vol 2. New Delhi: INBAR; 1996: p. 1-22.

29. Huberman MA. Bamboo silviculture. Unasylva. 1959;13(1):36-43.

30. Wang D, Shen S-J. Bamboos of China. Portland, Oregon: Timber Press; 1987.

31. Ram N, Singh L, Kumar P. Bamboo plantation diversity and its economic role in north Bihar, India. Nat Sci. 2010;8(11):111-5.

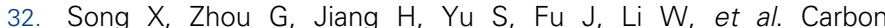
sequestration by Chinese bamboo forests and their ecological benefits: assessment of potential, problems, and future challenges. Environ Rev. 2011;19(1):418-28
33. Ohrnberger D. The bamboos of the world: annotated nomenclature and literature of the species and the higher and lower taxa. Amsterdam: Elsevier; 1999

34. Naithani HB. Diversity of Indian bamboos with special reference to north-east India. Indian For. 2008;134(6):765-88.

35. Biswas SAS. Studies on bamboo distribution in north-eastern region of India. Indian For. 1988;114(9):514-31.

36. Rai SN, Chauhan KVS. Distribution and growing stock of bamboos in India. Indian For. 1998;124(2):89-98.

37. Srivastava G, Su T, Mehrotra RC, Kumari P, Shankar U. Bamboo fossils from Oligo-Pliocene sediments of northeast India with implications on their evolutionary ecology and biogeography in Asia. Rev Palaeobot Palynol. 2019;262:17-27.

38. Choudhury D, Sahu JK, Sharma GD. Bamboo shoot: microbiology, biochemistry and technology of fermentation - a review. Indian $J$ Tradit Knowl. 2012;11(2):242-9.

39. Kaushik S, Singh YP, Kumar D, Thapliyal M, Barthwal S. Bamboos in India. Dehradun: ENVIS Centre on Forestry; 2015

40. Liese W, Welling J, Tang TKH. Utilization of bamboo. In: Liese W, Köhl M, editors. Bamboo: the plant and its uses. Cham: Springer; 2015. p. 299-346.

41. Gopalan C, Rama Sastri B V, Balasubramanian SC. Nutritive value of Indian foods. Hyderabad: ICMR; 1971.

42. Yamaguchi M. World vegetables: principles, production and nutritive values. Connecticut: Avi; 1983.

43. Bhargava A, Kumbhare V, Srivastava A, Sahai A. Bamboo parts and seeds for additional source of nutrition. J Food Sci Technol. 1996;33(2):145-6.

44. Tripathi YC. Food and nutrition potential of bamboo. MFP News $1998 ; 8(1): 10-1$

45. Bhatt BP, Singha LB, Singh K, Sachan MS. Commercial edible bamboo species and their market potentiality in three Indian triba states of the north eastern Himalayan region. J Bamboo Ratt. 2003;2(2):111-33.

46. Bhatt BP, Singh K, Singh A. Nutritional values of some commercia edible bamboo species of the north eastern Himalayan region, India. J Bamboo Ratt. 2005;4(2):111-24.

47. Nirmala C, David E, Sharma ML. Changes in nutrient components during ageing of emerging juvenile bamboo shoots. Int J Food Sci Nutr. 2007;58(8):612-8.

48. Kumbhare $\mathrm{V}$, Bhargava $\mathrm{A}$. Effect of processing on nutritional value of central Indian bamboo shoots. Part-1. J Food Sci Technol. 2007;44(1):29-31.

49. Nirmala C, Sharma ML, David E. A comparative study of nutrient components of freshly harvested, fermented and canned bamboo shoots of Dendrocalamus giganteus Munro. Bamboo Sci Cult J Am Bamboo Soc. 2008;21(1):41-7.

50. Ong HG, Kim Y-D. The role of wild edible plants in household food security among transitioning hunter-gatherers: evidence from the Philippines. Food Secur. 2017;9(1):11-24

51. Robinson AN, Scully C. Pharmacology: a new bleeding issue. $\mathrm{Br}$ Dent J. 2014;217(2):54-5.

52. Hwang IM, Yang J-S, Kim SH, Jamila N, Khan N, Kim KS, et al. Elemental analysis of sea, rock, and bamboo salts by inductively coupled plasma-optical emission and mass spectrometry. Anal Lett. 2016;49(17):2807-21.

53. Lee Y-C, Lee M-S, Jang S-J. Jukyeom as the source of trace elements to the human body: an analysis using In-san Jukyeom. J Food Sci Nutr. 2016;2(011):1-7.

54. Recht C, Wetterwald MF. Bamboos. London: BT Batsford; 1992 
55. Ghorbani A, Naghibi F, Mosaddegh M. Ethnobotany, ethnopharmacology and drug discovery. Iran J Pharm Sci. 2006;2(2):109-18.

56. Jain SK. Ethnobotany-its scope and various subdisciplines. In: Jain SK, editors. Manual of ethnobotany. 2nd ed. Jodhpur: Scientific Publishers; 1987. p. 1-11.

57. Ford RI. The nature and status of ethnobotany. Michigan: UMMAA 1978.

58. Heinrich M, Barnes J, Gibbons S, Williamson EM. Fundamentals of pharmacognosy and phytotherapy. Edinburgh: Churchill Livingstone; 2004.

59. Fongod AGN, Modjenpa NB, Veranso MC. Ethnobotany of Acanthaceae in the mount Cameroon region. J Med Plants Res. 2013;7(38):2859-66

60. World Health Organization, International Union for Conservation of Nature and Natural Resources, World Wide Fund for Nature. Guidelines on the conservation of medicinal plants. Gland: International Union for Conservation of Nature and Natural Resources; 1993.

61. Insoll T. Substance and materiality? the archaeology of Talensi medicine shrines and medicinal practices. Anthropol Med. 2011;18(2):181-203

62. Alves RRN, Rosa IL. Animals in traditional folk medicine. SpringerVerlag Berlin Heidelberg; 2013.

63. Hanif A, Hossan MS, Mia MMK, Islam MJ, Jahan R, Rahmatullah M. Ethnobotanical survey of the Rakhain tribe inhabiting the Chittagong Hill Tracts region of Bangladesh. Am J Sustain Agric. 2009:3(2):172-80.

64. Rahmatullah M, Biswas KR. Traditional medicinal practices of a Sardar healer of the Sardar (Dhangor) community of Bangladesh. J Altern Complement Med. 2012;18(1):10-9.

65. Rudra S, Islam KN, Rahman MM, Uddin SB. Medicinal plant diversity and their therapeutic uses in selected village common forests in Chittagong Hill Tracts, Bangladesh. J Herbs, Spices Med Plants. 2021;27(1):83-107.

66. Sharkar P, Rahman MM, Haque Masum GZ, Nayeem MA, Hossen MM, Azad AK. Ethnomedicinal importance of the plants in villages in Kushtia Sador and Mirpur Upozila, Bangladesh. J Herbs, Spices Med Plants. 2013;19(4):401-17.

67. Chassagne F, Deharo E, Punley H, Bourdy G. Treatment and management of liver diseases by Khmer traditional healers practicing in Phnom Penh area, Cambodia. J Ethnopharmacol. 2017:202:38-53.

68. Long C, Liu Y, Xue J, Li D. A bamboo germplasm collection for community development in central Yunnan, China. J Bamboo Ratt. 2003;2(1):3-11.

69. Luo B, Ahmed S, Long C. Bamboos for weaving and relevant traditional knowledge in Sansui, southwest China. J Ethnobiol Ethnomed. 2020;16:1-9.

70. Wang K, Hong LT, Rao VR. Diversity and indigenous utilization of bamboo in Xishuangbanna, Yunnan province, southwest China. $J$ Bamboo Ratt. 2002;1(3):263-73.

71. Yang YM, Wang KL, Pei SJ, Hao JM. Bamboo diversity and traditional uses in Yunnan, China. Mt Res Dev. 2004;24(2):157-65.

72. Anbarashan $M$, Parthasarthy $N$, Padmavathy A. Ethno-floristic survey in sacred groves, Pudukottai district, Tamil Nadu- India. J Med Plant Res. 2011;5(3):439-43.

73. Ayyanar $M$, Ignacimuthu $S$. Ethnobotanical survey of medicinal plants commonly used by Kani tribals in Tirunelveli hills of western Ghats, India. J Ethnopharmacol. 2011;134(3):851-64.
74. Bhatia H, Sharma YP, Manhas RK, Kumar K. Ethnomedicinal plants used by the villagers of district Udhampur, J\&K, India. J Ethnopharmacol. 2014;151(2):1005-18.

75. Esakkimuthu S, Mutheeswaran S, Arvinth S, Paulraj MG, Pandikumar $\mathrm{P}$, Ignacimuthu $\mathrm{S}$. Quantitative ethnomedicinal survey of medicinal plants given for cardiometabolic diseases by the noninstitutionally trained Siddha practitioners of Tiruvallur district, Tami Nadu, India. J Ethnopharmacol. 2016;186:329-42.

76. Jagtap SD, Deokule SS, Bhosle SV. Some unique ethnomedicinal uses of plants used by the Korku tribe of Amravati district of Maharashtra, India. J Ethnopharmacol. 2006;107(3):463-9.

77. Jain R, Jain SK. Traditional medicinal plants as anticancer agents from Chhattishgarh, India: an overview. Int J Phytomedicine. 2010;2(3):186-96.

78. Jain A, Katewa SS, Galav PK, Sharma P. Medicinal plant diversity of Sitamata wildlife sanctuary, Rajasthan, India. J Ethnopharmacol. 2005;102(2):143-57.

79. Singh V, Pandey RP. Ethnobotany of Rajasthan (India). Jodhpur: Scientific Publishers; 1998.

80. Jeeva S, Femila V. Ethnobotanical investigation of Nadars in Atoor village, Kanyakumari district, Tamilnadu, India. Asian Pac J Trop Biomed. 2012;2 Suppl 2:S593-600

81. Johnsy G, Davidson Sargunam S, Kaviyarasan V. Indigenous knowledge of medicinal plants used for the treatment of skin diseases by the Kaani tribal of Kanyakumari district. Int $\mathrm{J}$ Pharm Pharm Sci. 2012:4(1):309-13.

82. Majumder J, Bhattacharjee PP, Datta BK, Agarwala BK. Ethnomedicinal plants used by Bengali communities in Tripura, northeast India. J For Res. 2014;25(3):713-6.

83. Medhi P, Borthakur SK, Hore DK. Phytoresources from north Cachar hills of Assam, India-IV: bamboos and rattans. J Bamboo Ratt. 2010;9(3-4):115-25.

84. Prabhu S, Vijayakumar S, Morvin Yabesh JE, Prakashbabu R, Murugan R. An ethnobotanical study of medicinal plants used in Pachamalai hills of Tamil Nadu, India. J Herb Med. 2021;25:1-15.

85. Sathiyaraj K, Sivaraj A, Thirumalai T, Senthilkumar B. Ethnobotanical study of antifertility medicinal plants used by the local people in Kathiyavadi village, Vellore district, Tamilnadu, India. Asian Pac J Trop Biomed. 2012;2 Suppl 3:S1285-8.

86. Savithramma N, Sulochana C, Rao KN. Ethnobotanical survey of plants used to treat asthma in Andhra Pradesh, India. J Ethnopharmacol. 2007;113(1):54-61.

87. Sharma UK, Pegu S, Hazarika D, Das A. Medico-religious plants used by the Hajong community of Assam, India. J Ethnopharmacol. 2012;143(3):787-800.

88. Sharma TP, Borthakur SK. Ethnobotanical observations on bamboos among Adi tribes in Arunachal Pradesh. Indian J Tradit Knowl. 2008;7(4):594-7.

89. Silambarasan R, Ayyanar M. An ethnobotanical study of medicinal plants in Palamalai region of eastern Ghats, India. J Ethnopharmacol. 2015; 172:162-78.

90. Singh RAJ, Upadhyay SK, Rani A, Kumar P, Kumar A. Ethnobotanical study of Subhartipuram, Meerut, Uttar Pradesh, India. II. Diversity and pharmacological significance of shrubs and climbers. Int $J$ Pharm Res. 2020;12(2):383-93.

91. Singh B, Borthakur SK, Phukan SJ. A survey of ethnomedicinal plants utilized by the indigenous people of Garo hills with special reference to the Nokrek biosphere reserve (Meghalaya), India. J Herbs, Spices Med Plants. 2014;20(1):1-30.

92. Singh HB, Kumar B, Singh RS. Bamboo resources of Manipur: an overview for management and conservation. J Bamboo Ratt. 2003;2(1):43-55. 
93. Sukumaran S, Brintha TSS, Subitha P, Sheebha YA, Jeeva S. Usage of medicinal plants by two cultural communities of Kanyakumari district, Tamilnadu, south India. J Chem Pharm Res. 2014;6(8):6779 .

94. Sundriyal RC, Upreti TC, Varuni R. Bamboo and cane resource utilisation and conservation in the Apatani plateau, Arunachal Pradesh, India: implications for management. J Bamboo Ratt. 2002;1(3):205-46.

95. Udayan PS, George S, Tushar KV, Balachandran I. Some common medicinal plants used by the Nayaka community, Savandurga forest of Magadi taluk, Bangalore rural district, Karnataka, India. J Nat Remedies. 2005;5(1):35-40.

96. Jadid N, Kurniawan E, Himayani CES, Prasetyowati I, Purwani KI, Muslihatin W, et al. An ethnobotanical study of medicinal plants used by the Tengger tribe in Ngadisari village, Indonesia. PLoS One. 2020;15(7):1-16.

97. Jaya AM, Musa $Y$, Iswoyo H, Asmi N, Siregar LF, editors. Ethnobotanical study and identification of medicinal plants based on local knowledge. Proceedings of the 1st International Conference of Interdisciplinary Research on Green Environmental Approach for Sustainable Development; 2019 Aug 3-4; Universitas Muhammadiyah Buton, Indonesia. Bristol: IOP Conference Series: Earth and Environmental Science; 2019.

98. Novaryatiin S, Indah I. The medicinal plants used in Anjir Pulang Pisau, central Kalimantan-Indonesia. Pharmacogn J. 2019;11(6):1572-9.

99. Partasasmita R, An'Amillah A, Iskandar J, Mutaqin AZ, Annisa, Ratningsih N. Karangwangi people's local knowledge of bamboo and its role: implications for management of cultural keystone species. Biodiversitas. 2017;18(1):275-82.

100. Sujarwo W, Arinasa IBK, Peneng IN. Potensi bambu tali (Gigantochloa apus J.A. \& J.H. Schult. Kurz) sebagai obat di Bali. Bul. Littro. 2010;21(2):129-37.

101. Wulandari AP, Erick P, Joko K. Bambu AR-EE (Argumented RealityEnvironmental Education). Sumedang: Unpad Press; 2013.

102. Setiawati T, Mutaqin AZ, Irawan B, An'Amillah A, Iskandar J. Species diversity and utilization of bamboo to support life's the community of Karangwangi village, Cidaun sub-district of Cianjur, Indonesia. Biodiversitas. 2017;18(1):58-64.

103. Arinasa IBK. Bamboo diversity and utilization in Balinese rituals at Angsri Village-Bali, Indonesia. Bamboo Sci Cult J Am Bamboo Soc. 2010;23(1):29-37.

104. Utami NWF, Pradnyawathi NLM, editors. Diversity and utilization of bamboo plants in the area of hotel in Kedewatan village, Ubud, Bali. Proceedings of the 2nd International Symposium for Sustainable Landscape Development; 2016 Nov 9-10; Bogor, Indonesia. Bristol: IOP Conference Series: Earth and Environmental Science; 2017.

105. Kim H, Song M-J. Analysis and recordings of orally transmitted knowledge about medicinal plants in the southern mountainous region of Korea. J Ethnopharmacol. 2011;134(3):676-96.

106. Song M-J, Kim H, Lee B-Y, Brian H, Park C-H, Hyun C-W. Analysis of traditional knowledge of medicinal plants from residents in Gayasan National Park (Korea). J Ethnobiol Ethnomed. 2014:10:1-47.

107. de Boer HJ, Lamxay V, Björk L. Steam sauna and mother roasting in Lao PDR: practices and chemical constituents of essential oils of plant species used in postpartum recovery. BMC Complement Altern Med. 2011;11:1-10.

108. Libman A, Bouamanivong S, Southavong B, Sydara K, Soejarto DD. Medicinal plants: an important asset to health care in a region of central Laos. J Ethnopharmacol. 2006;106(3):303-11.
109. Awang-Kanak F, Abu Bakar MF, Mohamed M, Norazlimi NA, editors. Utilization of natural resources: preliminary study on ethnopharmacological application of "ulam" or traditional vegetables among Sama-Bajau of Kampung Menunggui, Kota Belud, Sabah. Proceedings of the International Conference on Applied Science and Technology; 2018 April 10-12; Penang, Malaysia. Maryland: AIP Conference Proceedings; 2018

110. Abe R, Ohtani K. An ethnobotanical study of medicinal plants and traditional therapies on Batan Island, the Philippines. J Ethnopharmacol. 2013;145(2):554-65.

111. Ong HG, Kim Y-D. Herbal therapies and social-health policies: indigenous Ati Negrito women's dilemma and reproductive healthcare transitions in the Philippines. Evidence-based Complement Altern Med. 2015;2015:1-13.

112. Neamsuvan O, Phumchareon T, Bunphan W, Kaosaeng W. Plant materials for gastrointestinal diseases used in Chawang district, Nakhon Si Thammarat province, Thailand. J Ethnopharmacol. 2016;194:179-87.

113. Phumthum M, Balslev H. Anti-infectious plants of the Thai Karen: a meta-analysis. Antibiotics. 2020;9(6):1-15.

114. Srisawat T, Suvarnasingh A, Maneenoon K. Traditional medicinal plants notably used to treat skin disorders nearby Khao Luang mountain hills region, Nakhon Si Thammarat, southern Thailand. J Herbs, Spices Med Plants. 2016;22(1):35-56

115. Sangeetha R, Diea YKT, Chaitra C, Malvi PG, Shinomol GK. The amazing bamboo: a review on its medicinal and pharmacological potential. Indian J Nutri. 2015;2(1):1-7.

116. Nirmala C, Bisht MS, Bajwa HK, Santosh O. Bamboo: a rich source of natural antioxidants and its applications in the food and pharmaceutical industry. Trends Food Sci Technol. 2018;77:91-9.

117. Shukla R, Sumit G, Sajal S, Dwivedi PK, Mishra A. Medicinal importance of bamboo. Int J Biopharm Phytochem Res. 2012;1(1):9-15.

118. Wróblewska KB, de Oliveira DCS, Grombone-Guaratini MT, Moreno PRH. Medicinal properties of bamboos. In: Perveen S, Al-Taweel A, editors. Pharmacognosy: medicinal plants. London: IntechOpen; 2019. p. 159-76.

119. Chongtham N, Bisht MS, Haorongbam S. Nutritional properties of bamboo shoots: potential and prospects for utilization as a health food. Compr Rev Food Sci Food Saf. 2011;10(3):153-68.

120. Suresh Kumar P, Usha Kumari K, Preema Devi M, Choudhary VK, Sangeetha A. Bamboo shoot as a source of nutraceuticals and bioactive compounds: a review. Indian J Nat Prod Resour. 2017;8(1):32-46.

121. Eisenberg DM, Davis RB, Ettner SL, Appel S, Wilkey S, Van Rompay $M$, et al. Trends in alternative medicine use in the United States, 1990-1997: results of a follow-up national survey. J Am Med Assoc. 1998;280(18):1569-75.

122. Sirven JI. Alternative therapies for seizures: promises and dangers. Semin Neurol. 2007;27(4):325-30

123. Mehrotra R, Bajaj S, Kumar D. Use of complementary and alternative medicine by patients with diabetes mellitus. Natl Med J India. 2004;17(5):243-5.

124. Li X-Z, Zhang S. Effervescent granules prepared using Eucommia ulmoides Oliv. and moso bamboo leaves: hypoglycemic activity in HepG2 cells. Evidence-based Complement Altern Med. 2016;2016:1-6

125. Nirmala C, Bisht MS, editors. Bamboo: a prospective ingredient for functional food and nutraceuticals. Proceedings of the 10th World Bamboo Congress; 2015 Sept 17-22; Damyang, Korea. Damyang: 10th World Bamboo Congress; 2015. 


\section{GRAPHICAL ABSTRACT}

\section{ETHNOBOTANY AND TRADITIONAL KNOWLEDGE OF BAMBOOS (POACEAE: BAMBUSOIDEAE) IN ASIA AND THEIR APPLICATIONS IN \\ THE COMPLEMENTARY AND ALTERNATIVE MEDICINE: A REVIEW}

\section{POACEAE}

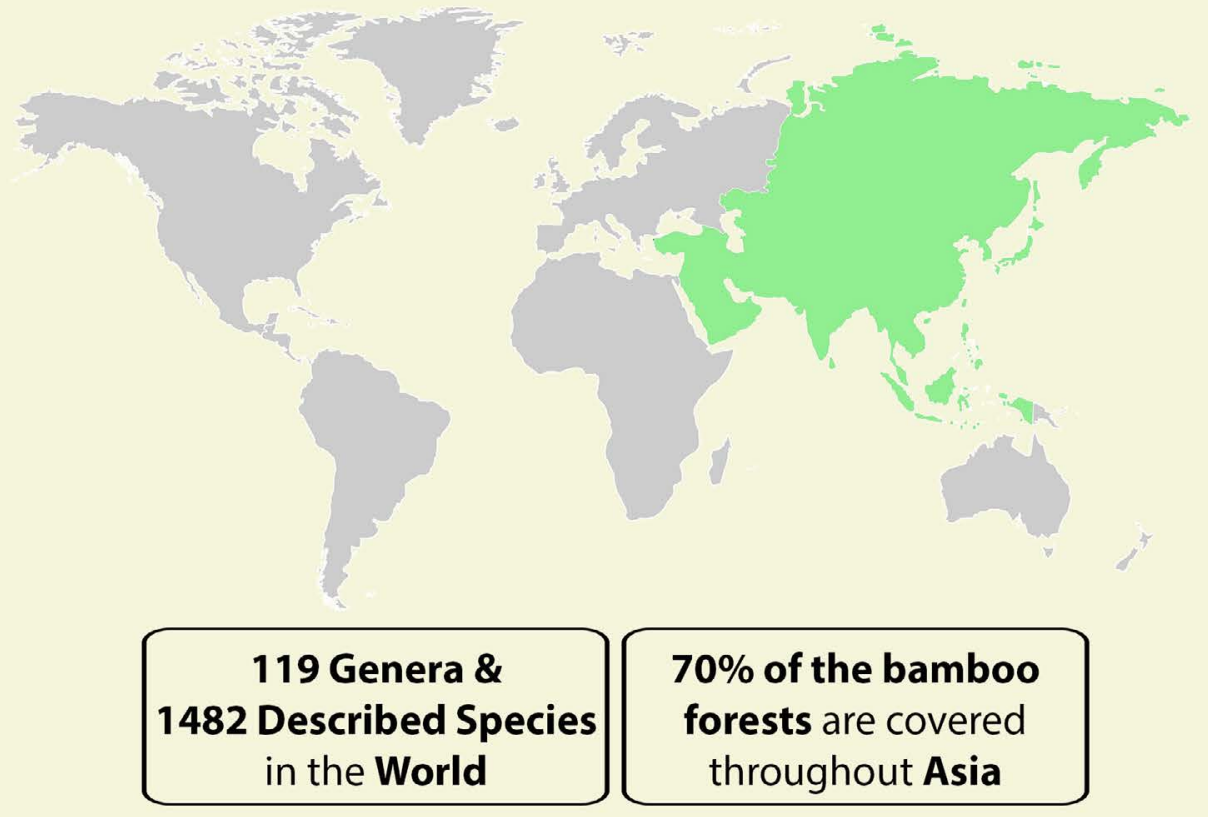

\section{REVIEW COVERS ON}

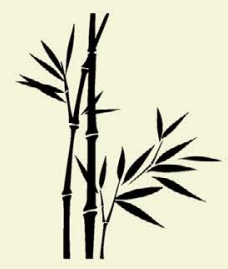

Part of the Plant Used

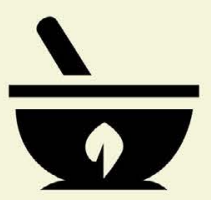

Application of Treatment

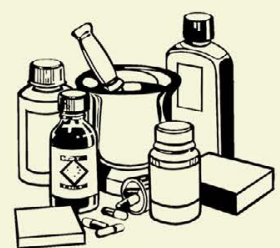

Complementary and Alternative Medicine (CAM)

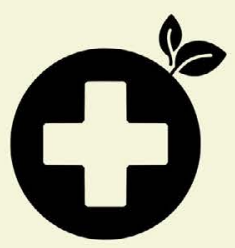

Ailment Treated 


\section{ABOUT AUTHORS}

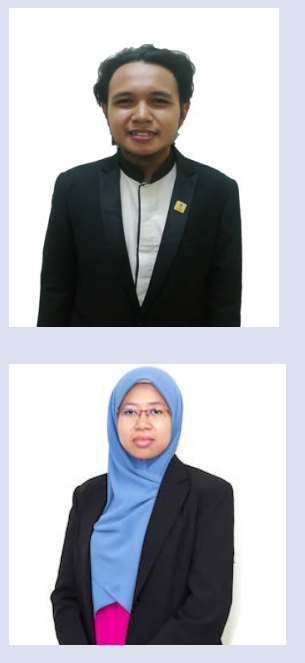

Mohammad Amil Zulhilmi Benjamin studied at Universiti Tun Hussein Onn Malaysia, taking a Bachelor of Science (Biodiversity and Conservation) with Honors. Now, he is pursuing his studies for a Master of Science (Advancement of Biodiversity) at Universiti Malaysia Sabah. His current expertise is in the fields of natural product chemistry, ethnobotany, and product development. He is also active as a researcher and has published several articles.

Dr. Nor Azizun Rusdi is a Senior Lecturer at the Institute for Tropical Biology and Conservation, Universiti Malaysia Sabah. She obtained her bachelor's degree in Biochemistry at Universiti Putra Malaysia. She got her Master of Science in Plant Biotechnology from Universiti Putra Malaysia. She further pursued her doctoral degree in Systems Biology at Universiti Kebangsaan Malaysia. She is currently executing her research project in plant tissue culture of wild orchids, plant functional genomics, and natural product research from medicinal plants.

Dr. Fiffy Hanisdah Saikim is a Senior Lecturer at the Institute for Tropical Biology and Conservation, Universiti Malaysia Sabah. She graduated with a Bachelor of Science (Science and Aquatic Resource Management) with Honors from Universiti Malaysia Sarawak, Master of Science (Nature Tourism) from Universiti Malaysia Sabah in Malaysia, and Doctor of Philosophy (Tourism) from James Cook University, Australia. Her research interests include human-wildlife conflicts, environmental education, development of nature-based tourism products, Satoyama Landscape, community-based conservation and tourism, entomological ecotourism, and community empowerment.

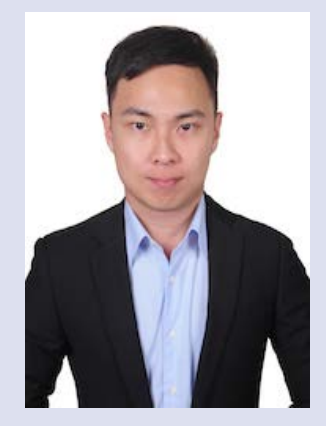

Dr. Ng Shean Yeaw is a Senior Lecturer at the Institute for Tropical Biology and Conservation, Universiti Malaysia Sabah. He obtained his bachelor's degree in Conservation Biology from Universiti Malaysia Sabah. He was enrolled in the fast-track program at Universiti Malaysia Sabah for a PhD degree in Advancement of Biodiversity. His research interest is in the phytochemical and bioactive potential of Bornean liverworts, and indigenous fruits and vegetables.

Cite this article: Benjamin MAZ, Ng SY, Saikim FH, Rusdi NA. Ethnobotany and Traditional Knowledge of Bamboos (Poaceae: Bambusoideae) in Asia and Their Applications in the Complementary and Alternative Medicine: A Review. Pharmacogn J. 2021;13(6) Suppl: 1751-1762. 